\title{
Narraciones orales y escritas en niños. \\ Un estudio sobre sus diferencias
}

\author{
R. A. CLEMENTE \\ Universidad de Málaga
}

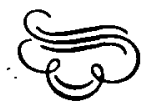

Resumen

El objetivo de este trabajo fue comprobar las diferencias existentes entre las modalidades oral y escrita de expresión. El tipo discursivo elegido para la comparación fue la narración espontánea, fundamentalmente por la literatura psicológica y psicolingüistica que la apoya. Se analizaron 64 narraciones, 32 orales y 32 escritas de otros tantos sujetos todos ellos alumnos - del último trimestre de primer curso de E.G.B. Las variables analizadas fueron nivel narrativo, cohesión y presencia de marcadores, M. L. E., cantidad de palabras, cantidad y tipo de nexos, cantidad y tipo de formas verbales.

Los resultados parecen demostrar que es más lo que une que lo que separa a ambas modalidades si no existe a la vez de la comparación intermodal la comparación interdiscursiva. Así no resultan diferentes significativamente más que los marcadores narrativos (las medidas más altas son de la modalidad escrita) y la productividad del texto, (las medidas más altas cuantitativamente son de la modalidad oral); el resto de variables no son significativamente diferentes.

\section{Summary}

The aim of this paper is to test differences between oral and writen modalities of expresion. The discoursive type taken for the comparison was spontaneus narration, because of its psychological and psycholinguistic literature support. 64 narrations were analyzed, 32 oral and 32 writen, by the same number of subjets, all of them in the last term of the lest. course of E.G.B. The analyzed variable were narrative level, cohesion and use of markers, M. L. U., number of words, number and type of verbal forms.

Results seem to show that both modalities has much in common if interdiscursive comparison in not added to the intermodal one. Thus, there are not significative differences between narrative markers (The bighest measure were in the writen modality) and text productivity (the highest measures were in the oral modality). No other variables was significatively different.

Dirección de la autora: Universidad de Málaga. Facultad de Filosofía y Letras. Departamento de Psicología. Edificio del Rectorado, campus El Ejido, 29013 Málaga. 
Parece ser propio de la investigación reciente el cuestionar o al menos matizar una creencia muy generalizada en ámbitos psicopedagógicos, acerca de la continuidad entre las formas orales y escritas de expresión del habla (Benveniste, 1982; Smith, 1975).

No se trata de renunciar a lo que realmente une a ambas modalidades (sistemas de expresión, relación de significantes-significados, forma de ejecución del pensamiento, etc.), sino de especificar que la línea de continuidad entre ambas no es una adición sin más de elementos estructuralmente cada vez más complicados; para los hablantes ambos tipos de formas discursivas tienen diferente valor y, por tanto, existen diferencias en su uso y construcción, por lo que cabría esperar que también en su aprendizaje.

Algunos autores parecen decantarse por el continuum entre ambas modalidades (Danielewicz, 1984); según esta opción, lenguaje hablado y escrito se reconocerían como formando parte del mismo eje; en él, situado en su extremo inferior se situaría el discurso espontáneo oral y en su extremo superior el discurso escrito con intención expositiva.

Sin embargo, la mayoría de los autores consultados destacan las diferencias entre ambos. Hemos recogido especialmente argumentos que las centran en:

a) El lenguaje hablado está muy contextualizado, mientras que el escrito es descontextualizado (Olson, 1977; Collins y Williamson, 1981).

b) La coherencia en el discurso hablado se consigue mediante conductas paralingüísticas (entonación, prosodia, etc.), comunicación no-verbal (expresión facial, gestos, etc.) y elementos cognitivos (como las presuposiciones, etc.); mientras que la cohesión en el discurso escrito es lingüística (relación entre proposiciones, encadenamiento de frases, signos de puntuación, etc.) (Chafe, 1980; Cook-Gumperez y Corsaro, 1977; Bates, 1976).

c) El discurso oral es ágil y fragmentado, el escrito formal es organizado, compacto e integrado. Por ejemplo, y a consecuencia de esto, C.B. Benveniste le ha llamado «lenguaje de domingos». Está, asimismo, comprobado que el estilo al escribir es menos redundante y léxicamente más variado (Schafer, 1981).

El hecho de que el aprendizaje del lenguaje oral sea informal, se lleve a cabo en el hogar y a través de la interacción, mientras que el del escrito sea cultural, formal y, por tanto, escolar, apoya los bloques de diferencias vistas, y no hace, por otro lado, sino ratificar la opinión de quienes piensan que los niños al aprender a leer y escribir deben aprender algo nuevo, deben reconstruir la modalidad interactivo-comunicativa oral en función de otra formal, independiente, descontextualizada, que tiene menos valor comunicativo, pero más funcionalidad cultural.

Incluso en idiomas tan fonéticos como el castellano, los niños parecen tener, en general, serias dificultades en escribir su discurso. Nuevas exigencias se suman a la hora de plasmar por escrito algo que se desea decir. Los niños, acostumbrados durante su infancia a una modalidad que mantiene el vínculo contextual y que está bajo la presencia temporal y espacial de otro interlocutor, deben pasar a usar el lenguaje sin interlocutor, con posibilidad de tener vigencia por sí mismo, que incluye todo tipo de recursos para su comprensión sin la presencia de ningún contexto concreto.

Cuando los niños se enfrentan con las modalidades escritas de los dis- 
cursos, están ya en períodos escolares (obligatorios o no) y su destreza en discursos orales es importante, pero su costumbre también; ellos deben adaptar una modalidad muy abierta (por lo interactiva y contextual) de discurso a otra modalidad más cerrada, independiente y que exige registro físico y respetuoso cumplimiento de sus propias normas.

Hemos intentado realizar una investigación comparando ambas modalidades de expresión, utilizando un discurso acotado: la narración, con amplia tradición en su análisis (Mandler y Johnson, 1977; Johnson y Mandler, 1980; Stein y Glen, 1979; Marchesi, 1981), pretendiendo de esta forma establecer sistemas de medida que permitan la comparación y que garanticen cierta coherencia intra-discurso; además, equiparamos al máximo la variabilidad existente inter-modalidades al mantener una macroestructura narrativa igual para ambas modalidades.

El tipo discursivo elegido ha sido la narración, fundamentalmente por sus características estructurales, ya que permite su división en unidades que se organizan secuencialmente desde los marcadores iniciales hasta la resolución de los conflictos, y por tanto, su final (Stein y Glen, 1979). También los datos empiricos demuestran que es uno de los discursos en el que los niños obtienen resultados más precoces (Sutton-Smith, Botvin y Mahoney, 1976; Clemente, 1983).

\section{MÉTODO}

Hemos recogido ejemplos de narraciones orales y escritas en treinta y dos sujetos de seis/siete años. Los niños estaban en el último trimestre de primer curso de E.G.B. y todos fueron capaces de escribir y de contar oralmente un cuento. Se eliminaron dos sujetos cuya incapacidad para escribir les imposibilitaba para la prueba.

Las narraciones pueden considerarse espontáneas, puesto que se solicitó su emisión a los sujetos sin consideraciones previas, tan sólo bajo la indicación general de "contar un cuento». Para evitar que los niños repitieran cuentos tradicionales o cuentos inventados por otras personas, se acordó dar algunas directrices que obligaran a construir o inventar un cuento en el momento.

Así, para la narración escrita, tras dar una hoja en blanco, lápiz y goma, se dijo a los niños que escribieran un cuento en el que aparecieran tres objetos (un gato, un ratón y un niño); en el momento de la orden, el experimentador mostraba tres objetos representativos de pequeño tamaño. Las narraciones escritas se pasaron colectivamente; se permitió a los niños borrar y usar todo el tiempo que deseasen.

Las narraciones orales se pasaron individualmente y con registros magnetofónicos simultáneos; como en la otra prueba, para evitar narraciones al uso, también se dirigió la narración de un cuento espontáneo por la exigencia de la participación en el tema de algunos objetos, en este caso: un muñeco, un oso y un plato. Los tres objetos permanecían sobre la mesa y se permitía al niño su manipulación si lo deseaba.

De cada narración se han contabilizado las siguiente variables:

De carácter «macroestructural», referidas a la organización global del discurso, en este caso narrativo: 
Marcadores del inicio y/o del final de la narración, es decir la presencia o no de expresiones del tipo "había una vez", "este cuento se acabó», "colorín colorado...", etc.

Complejidad estructural de las narraciones (siguiendo con modificaciones las directrices marcadas por Botvin y Sutton-Smith, 1977). Hemos utilizado las tipologías descritas por estos autores, puesto que su investigación, como la nuestra, hace referencia a narraciones inventadas por los niños.

La superestructura de la narración se describe habitualmente por los lingüistas como constituida por categorias semejantes a las siguientes: exposición, desarrollo, resolución, valoración y epílogo (Van Dijk, 1980); como estas categorías suelen estar precariamente o no representadas en las narraciones incipientes de los niños (Roth y Spekman, 1986) hemos considerado las descritas por Sutton-Snith y Botvin, que simplifican semánticamente las referencias narrativas, siguiendo la tradición del antropólogo ruso Propp (1977).

En esta tradición científica se consideran categorías fundamentales para decidir si un discurso es o no narración, las siguientes:

A) Aparición de algunos personajes destacados que mantengan su protagonismo durante un número de expresiones.

B) Que estos personajes tengan ocasión de iniciar un proceso argumentativo de narración (una de las llamadas diadas argumentativas), que pueden ser de dos tipos:

1. Diada de maldad, con la aparición de un personaje en el papel del «villano» que realiza una maldad. La segunda parte de la diada se corresponde con su anulación o la maldad acabada.

2. Diada de carencia, cuando a un personaje le falta algo (amor, compañía, comida...) también se exige su subsanación o anulación mediante la solución de la carencia.

C) Para que haya narración, es necesario que entre las diadas, algunas expresiones estén dedicadas a la acción de la primera parte y a la reacción de la segunda.

D) Es necesario que la historia tenga un fin, sea o no convencional, es decir, que el proceso argumentativo se cierre.

Botvin y Sutton-Smith (1977) separan, siguiendo estas directrices, las narraciones infantiles en siete niveles, que nosotros hemos seguido y que para su mejor comprensión describimos con ejemplos en el apéndice.

Cohesión narrativa y coberencia de las expresiones del texto con el nudo narrativo descrito en la narración, en este caso se ha usado, modificado, el sistema de análisis descrito por Liles (1985). Hemos contabilizado las expresiones de la narración que contribuyen al desarrollo del hilo narrativo, y las hemos separado de aquellas que, aún estando en la narración, no contribuyen a él, ya sea porque inician un nudo nuevo que no llega a cumplirse, o porque son expresiones ajenas a la narración.

De carácter «microestructural» o aspecto dirigido a la complejidad sintáctica de los textos, hemos analizado:

Cantidad total de palabras. 
Cantidad total de expresiones. Entendiendo por expresión toda cadena hablada o escrita aislada por el silencio (más de dos segundos en la modalidad oral) o por cambio de tema (en la modalidad escrita) o por nexos coordinantes (en ambas modalidades).

M. L. E. (media de longitud de expresión).

Frecuencia de los diversos tipos de marcadores de complejidad sintáctica.

Frecuencia de los diversos tipos de complejidades verbales.

\section{RESULTADOS Y DISCUSIÓN}

Relativos a las variables macroestructurales.

\section{Marcadores del inicio y fin de la narración}

Hay diferencias importantes y significativas respecsto a la cantidad de marcadores del principio y final del cuento. Las cantidades registradas aparecen en la Tabla $\mathrm{I}$.

\section{TABLA I}

Distribución de los marcadores de inicio y final en las 64 narraciones

\begin{tabular}{lcccr}
\hline Presencia de marcador & ++ & -+ & +- & $-\bar{c}$ \\
Narrac. orales & I F & I F & I F & I F \\
Narrac. escritas & 1 & 3 & 13 & 15 \\
\hline
\end{tabular}

Los datos son significativos $\left(\chi^{2}=24,04\right)$ al 0,01 . Las narraciones escritas están en gran número (26/32) introducidas por una marca superficial típica; mientras que en las orales sólo cumplen este requisito 14 narraciones de las analizadas.

De igual forma, 19 de las treinta y dos narraciones escritas marcan explícitamente el final de la narración, siendo tan sólo cuatro de las orales las que lo hacen.

Los hechos no merecen más comentario que atribuir mayor formalidad a la modalidad escrita, por tanto los recursos superficiales se usan de forma más disciplinada en la modalidad escrita que en la oral.

\section{Los niveles narrativos.}

La tabla II resume los datos y su significación $\left(\chi^{2}=4,10\right)$ y por tanto no significativa.

\section{TABLA II}

Distribución de los niveles narrativos en las 64 narraciones

\begin{tabular}{|c|c|c|c|c|c|}
\hline Nivel & 1 & 2 & 3 & 4 & 5 \\
\hline $\begin{array}{l}\text { Oral } \\
\text { Escrito }\end{array}$ & $\begin{array}{l}13 \\
15\end{array}$ & $\begin{array}{l}3 \\
7\end{array}$ & $\begin{array}{l}7 \\
6\end{array}$ & $\begin{array}{l}5 \\
3\end{array}$ & $\begin{array}{l}4 \\
1\end{array}$ \\
\hline
\end{tabular}


Botvin y Sutton-Smith (1977) encontraron que entre 5 y 6 años los niños empezaban a demostrar su capacidad para producir narraciones estructuralmente completas. Sus datos coinciden con los de Applebee (1978) y de Clemente (1983). Ahora bien, la existencia de episodios y de jerarquización entre ellos sólo está completa hacia 11-12 años, sobre todo en tanto en cuanto se mide la inclusión de episodios en otros, es decir; la existencia de sub o supraepisodios.

Nuestras narraciones, tanto orales como escritas, mantienen y confirman las conclusiones de la literatura: nuestros niños llegan al nivel 5 , pero no a los jerarquizados 6 y 7 . Sin embargo las diferencias inter-tareas no son significativas. Se puede decir que la estructura de la narración apoya la organización productiva que le es propia, tanto si ésta se expresa bajo la modalidad oral como escrita.

Sin embargo, se nota la tendencia del lenguaje escrito a utilizar menos expresiones, lo que rebaja el nivel entre pares de estructuras con la misma complejidad pero menos acciones entre el inicio y el final, (por ejemplo entre el nivel 2 y el 3 , la única diferencia es que el segundo está formado por una sola secuencia y el tercero por muchas; igual sucede entre los niveles 4 y 5). Todo funciona como si efectivamente el lenguaje escrito llevara a los niños a terminar cuanto antes, poniendo lo esencial de la narración, mientras que su destreza en la expresión oral les lleva a incluir más personajes, más acciones, más reacciones y, por tanto, más episodios; sin embargo la estructura narrativa no por ello se ve mejorada sustancialmente (al menos significativamente).

Como puede verse en los ejemplos del apéndice, la simplicidad, y la carencia de ornato en las narraciones escritas es francamente notoria, frente a la prolijidad de las narraciones orales, sin que la estructura narrativa cambie de forma importante.

\section{Cobesión narrativa}

La cantidad de expresiones coherentes con el hilo narrativo dentro de cada una de las sesenta y cuatro narraciones analizadas ha sido tal y como se describe en la Tabla III. Si se comparan estas cantidades brutas eliminando los efectos que por la mayor longitud afectan a las narraciones orales, por ejemplo, haciendo porcentajes, resultan cantidades muy semejantes. Así, el $65,76 \%$ de coherencia en las expresiones escritas para con el nudo preferente y el $63,93 \%$ para las narraciones orales. Resultando de la comparación diferenciadas no significativas $\left(\chi^{2}=0,26\right)$.

TABLA III

Distribución de las expresiones en función de su cobesión

\begin{tabular}{lccc}
\hline \multicolumn{1}{c}{ Expresiones cohesión } & Con cohes. & Sin cohes. & Total \\
\hline Oral & 367 & 207 & 574 \\
Escrito & 171 & 89 & 260 \\
\hline
\end{tabular}




\section{Relativos a las variables microestructurales}

\section{Cantidad de elementos en las frases}

Como puede observarse en la Tabla IV, las narraciones orales son más largas, las diferencias en cantidad de palabras y de expresiones entre ambos tipos de modalidades son muy significativas; sin embargo, la cantidad relativa de palabras que hay dentro de cada expresión (M. L. E.) es muy similar y, por tanto, no significativa, lo que sin duda demuestra dos cosas: en primer lugar, que sólo se trata de una modalidad superficialmente más productiva, puesto que la relación palabras/expresiones no es diferente $y$, en segundo lugar, cómo la incipiente destreza del niño escribiendo no permite al discurso escrito alargar la cantidad de elementos dentro de las frases, ya que suele ser habitual que las M. L. E. en las modalidades escritas sean superiores a las orales.

TABLA IV

Medias y significación en palabras, expresiones $y$ M. L. E. Narraciones orales y escritas

\begin{tabular}{|c|c|c|c|c|}
\hline & Escrita & Oral & t & Sig. \\
\hline \multirow{3}{*}{$\begin{array}{l}\text { Cant. de palabras } \\
\text { Cant. de expresiones } \\
\text { Ce } \\
\text { M. L. } \frac{\dot{\bar{X}}}{\overline{\mathrm{X}}}\end{array}$} & 53,31 & 121,12 & 5,69 & 0,00 \\
\hline & 8,12 & 17,93 & 6,09 & 0,00 \\
\hline & 6,62 & 6,75 & 0,45 & 0,65 \\
\hline
\end{tabular}

Aunque alguna literatura considera la presencia de las complejidades sintácticas como índice de cohesión (Liles, 1985) y, de hecho, los autores analizan el tipo de oraciones usadas en relación con su valor semántico (Halliday y Hasan, 1976); en nuestro caso, dada la poca variabilidad de nexos y la ausencia de muchos de ellos, para no incrementar el número de casillas vacías, hemos agrupado las menos usadas de forma lingüísticamente coherente, dejando como categorías singulares las más especificas de la narración.

Los tipos de oraciones se han clasificado en cuatro grupos: el primero constituido por las tematizaciones o falsas relativas, muy características de las narraciones. Se trata de frases del tipo: "había una vez un $\mathrm{X}$ que...", o "era un $\mathrm{Y}$ que...»; el segundo constituido por las frases con la coordinante "y» y sus variantes «y luego», "y después», etc; el tercer grupo lo constituyen todas las frases subordinadas y el cuarto bloque todas las otras coordinadas. Las oraciones simples no se han contabilizado.

Los resultados de las comparaciones pueden observarse en la Tabla V. Las frecuencias se han transformado en índices con el fin de poder hacer las comparaciones, dada la mayor cantidad total de elementos dentro de la modalidad oral. En los índices se ha usado como denominador el número total de expresiones emitidas por cada niño. Así los datos correspondientes a las medias no son frecuencias, sino "índices de uso en función de las expresiones totales registradas". 


\section{4}

TABLA V

Indices medios y significación de los tipos de frases. Narraciones orales y escritas

\begin{tabular}{lcccc}
\hline & Escrita & Oral & t & Sig. \\
\hline Tematizaciones & 12,6 & 8,28 & 1,88 & 0,06 \\
$\begin{array}{c}\bar{X} \text { índice } \\
\text { Frases con «y» }\end{array}$ & 47,6 & 52,90 & 0,79 & 0,43 \\
$\begin{array}{c}\bar{X} \text { índice } \\
\text { Subordinadas } \\
\bar{X} \text { índice }\end{array}$ & 22,84 & 32,53 & 1,65 & 0,10 \\
Otras coord. & 19,28 & 16,46 & 0,77 & 0,44 \\
$\bar{X}$ índice & & & & \\
\hline
\end{tabular}

Ninguna de las diferencias son significativas al 0,05 ; si bien la diferencia entre tematizaciones da una significación de 0,06.

Parece, en este sentido, decantarse una direccionalidad del texto éscrito hacia un tipo de discurso narrativo más lleno de marcas superficiales narrativas que el oral, fundamentalmente por la presencia de falsas relativas. En cualquier caso, la gran cantidad de coordinante "y", demuestra en ambas modalidades que los hablantes son sujetos jóvenes que hacen descripciones de hechos muy propias, por otra parte, de las narraciones.

\section{Las formas verbales}

La variabilidad en las formas verbales constituye un aspecto característico de los tipos discursivos y según cuál sea éste, las acciones que se describen aparecen dentro de los tiempos verbales más idóneos para los propósitos macroestructurales del texto. En el discurso narrativo, como discurso que mantiene diferencias temporales entre el momento del tema a narrar y el tiempo de la enunciación, la presencia de tiempos del pasado es significativamente importante, como ha descrito Bronckart (1982).

En las narraciones escritas los pasados ocupan un $86,59 \%$ de todas las formas verbales que se registran, mientras que en las narraciones orales el porcentaje es de $72,91 \%$. Se puede decir que ambos en general, pero más afianzadamente el texto escrito, respetan la organización estructural de la narración.

La comparación entre ambas modalidades (de nuevo los datos son índices de las formas verbales en función de la cantidad de expresiones de cada narración), ofrece el panorama de la Tabla VI.

TABLA VI

Indice medios y significación de los tipos de formas verbales. Narraciones orales y escritas

\begin{tabular}{|c|c|c|c|c|}
\hline & Escrita & Oral & $\mathbf{t}$ & Sig. \\
\hline \\
\hline Tiempos de pasado & & & 2,07 &,, 0 \\
\hline$\overline{\mathrm{X}}$ & 99,12 & 90,65 & 1,06 & 0,29 \\
\hline Otras t..v. & 6,06 & 10,43 & 1,65 & 0,10 \\
\hline
\end{tabular}


La única diferencia casi significativa aparece en los tiempos del presente, las narraciones orales tienen más tiempos del presente; suponemos que atribuibles a la presencia del interlocutor y también a diálogos internos (directos) dentro de las narraciones, cosa prácticamente inexistente en las narraciones escritas, en las que el estilo en los diálogos es preferentemente indirecto.

\section{CONCLUSIONES}

De los ocho indicadores contabilizados para comparar, podríamos resumir las diferencias intermodalidades de la forma siguiente:

Respecto a la organización general narrativa, no hay diferencias significativas, salvo en los niveles más superficiales, como el de las marcas de inicio y final. Parece esperable que ante un papel en el que se debe plasmar algo planificado, la consigna de narrar, lleve a enmarcar el texto con su principio y su fin, aunque a veces no haya narración que estructuralmente pueda considerarse como tal.

La calificación narrativa respecto a su organización estructural, aunque ligeramente superior para el lenguaje oral, no resulta significativa. Los niños de seis/siete años son capaces en un 59,37 \% oralmente y en un 53,12\% escribiendo, de hacer narraciones en las que al menos un episodio se inicie y finalice, en las que algún personaje adopte papeles relevantes y lleve a cabo acciones y reacciones para resolver un argumento.

El resto de narraciones, las pertenecientes al nivel 1, no pueden considerarse propiamente como tales, puesto que son tan sólo el resultado de yuxtaposiciones únicamente relacionadas por acciones, por hechos que se suceden $\sin$ que se den núcleos narrativos a los que se subordinen los hechos.

La coherencia de las expresiones respecto al nudo narrativo es muy similar en ambos tipos de modalidades, nuestros sujetos tienen gran cantidad de expresiones (ver Tabla III) dedicadas a avalar la coherencia al texto narrativo, así como otras muchas irrelevantes en las que se cuentan hechos inexplicables o acciones semánticamente coherentes pero yuxtapuestas entre sí, sin respeto a una organización general; frecuentemente aparecen personajes o nudos nuevos dejando sin finalizar el anterior, cortando, en definitiva, el hilo narrativo anterior.

De cualquier forma, el hecho de que esto ocurra sin diferencias en ambas modalidades, hace pensar que se trata de un problema evolutivo-cognitivo (Winner, 1981) en gran medida independiente del modo de expresión.

Los indicadores más externos, referidos a la organización morfo-sintáctica, no mantienen diferencias significativas, si se exceptúa la longitud del discurso (cantidad de palabras y cantidad de expresiones), el cual, como cabría esperar, dada la mayor experiencia infantil, es mucho más fluido y largo para la modalidad oral. El resto de marcas respetan la organización narrativa sin que se establezcan diferencias significativas entre ambas modalidades.

Resulta cuanto menos interesante, comprobar cómo los datos contabilizados demuestran que ambas modalidades cumplen los requisitos lingüisticos que exigen las narraciones y descritos por los lingüistas y psicolin- 
güistas del texto (verbos en pasado, marcadores introductorios, etc.) (Bronckart, 1982; Weinrich, 1981), lo que sin duda demuestra que el tipo de discurso domina tanto la acción de hablar como la de escribir, independientemente de se escriba o se hable.

Según nuestros datos, las diferencias simplistas entre modalidades orales y escritas no puede plantearse, tal y como parece desprenderse de este trabajo, el tipo discursivo unifica las respuestas superando las diferencias intermodalidades. En esta línea otros autores también han argumentado en favor de otras variables distintas a la modalidad, como la función comunicativa, el contexto o el tipo discursivo. Autores como Scribner y Cole (1981), Rubin (1980), o Tannen (1982), reconocen que hay similitudes entre las expresiones orales y escritas si la función comunicativa o la intención discursiva son las mismas.

\section{Referencias}

APPLEBEE, A. N. (1978). The child's concept of story. Chicago: University of Chicago Press.

BATES, E. (1976). Language and context: the acquisition of pragmatics. Nueva York. Academic Press.

BENVENISTE, C. B. (1982). L'image de la norma linguistique prope aux textes écrits. Les différences entre cette norme et les normes de la langue cotidienne. Primeres Jornades sobre la representació escrita en el nen. Barcelona.

BENNETT-KASTOR, T. (1983). Noum phrases and coherence in child narratives. Journal of Child Language, 10, 135-149.

Botvin, G. J., y SUTTON-SMITH, B. (1977). The development of structural complexity in children's fantasy narratives. Developmental Psycbology, 13, 377-388.

BRONCKART, J. P. (1982). Classification de textes pour la redaction et l'analyse. Cabiers de la Section des Sciences de l'Education. Génova: Universidad de Génova.

Collins, J. L., y WILlIAMSON, M. W. (1981). Spoken language and semantic abbreviation in writing. Research in the Teaching of English, 15, 23-36.

COOK-GumperZ, J., y CORSARO, W. (1977). Social-ecological constraints on children's communicative strategies. Sociology, $11,411-434$.

CHAFE, W. L. (1980) (comp.). The pear stories: cognitive, cultural and linguistic aspects of narrative production. Norwood, N. J.: Ablex. Publ.

Clemente, R. A. (1983). Variaciones en las producciones espontáneas de los niños. Comunicación presentada en el IV Seminario sobre Investigaciones Actuales en Psicología Evolutiva y de la Educación. Madrid.

Clemente, R. A. (1984). Variaciones en el lenguaje espontáneo infantil. En M. Siguan (comp.): Estudios sobre psicología del lenguaje infantil. Madrid: Pirámide.

DANIELEwICZ, J. M. (1984). The interaction between text and context: A study of how adults and children use spoken and written languaje in four context. En A. Pellegrini y TH. Yawkey (comps.): The development of oral and written language in social context, Norwood, N. J.: Ablex. Publ.

VAN DijK, T. A. (1980). Story comprehension: An introduction. Poetics, 9, 1-121.

Halliday, M. A. K., y HASAN, R. (1976). Cobesion in english. Londres: Logman.

HudSON, J., y NELSON, K. (1983). Effects of script structure on children's story recall. Developmental Psychology, 19, 625-635.

JOHNSON, N. S., y MANDLER, J. M. (1980). A tale of two structures: underlying and surface forms in stories. Poetics, 9, 51-86.

LILES, B. Z. (1985). Production and comprehension of narrative discourse in normal and language disordered children. Journal of Communication Disorders, 6, 409-427.

MANDLER, J. M., y JOHNSON, N.S. (1977). Remembrance of things parsed: story structure and recall. Cognitive Psychology, 9, 111-151.

Marchesi, A. (1981). Memoria y desarrollo cognitivo. Anotaciones en torno a la teoría de Paiaget sobre la memoria. Infancia y Aprendizaje, 15, 91-109.

OlsoN, D. R. (1977). From utterance to text: the bias of language in speech and writing. Harvard Educational Review, 47, 257-281.

PELLEGRINI, A., Y YAWKEY, TH. (Ed.) (1984). The development of oral and written language in social contexts. Norwood, N. J.: Ablex. Publ.

Propp, V. (1977). Morfología del cuento. Madrid: Fundamentos. 
Roth, F. P., y SPEKMAN, N. J. (1986). Narrative discourse: spontaneously generated stories of learning-disabled and normally achieving students. Journal of Speech and Hearing disorders, si, 8-23.

RuBIN, A. (1980). A theoretical taxonomy between oral and written language. En R. J. Spiro, B. C. Bruce y W. F. Brewer (comps.): Theoretical issues in reading comprehension. Hillsdale, N. J.: Erlbaum.

SCHAFER, J. C. (1981). The linguistic analysis of spoken and written texts. En B. M. Kroll y R. J. Vann (comps.): Exploring speaking-writing relationships: conections and contrasts. Urbana: Il. NCTE.

SCRIBNER, S., y COLE, M. (1981). Unpackaging literacy. En M. F. Whiteman (comp.): Writing: The nature, development, and teaching of written communication: Vol. 1. Variation on writing: Functional and linguistic-cultural differences. Hillsdale, N. J.: Erlbaum.

SMITH, F. (1975). Comprebension and learning. Ontario Institute for Studies in Education. Ontario Hot, Rinehart and Winston.

STEIN, N. L., y GLENN, C. G. (1979). An analysis of story comprehension in elementary school children. En R. O. Freedle (comp.): New directions on discourse processing: Vol. 2. Norwood, N. J.: Ablex. Publ.

Sutton-Smith, B.; Botvin, G., y Mahoney, D. (1976). Developmental structures in fantasy narratives. Human Development, 19, 1-13.

TANNEN, D. (1982). Oral and literary strategies in spoken and written narratives. Languaje, $58,1-21$.

UMIKER-SEBEOK, D. J. (1979). Preschool children's intraconversational narratives. Journal of Child Language, 6, 91-109.

WEINRICH, H. (1981). Lenguage en textos. Madrid: Gredos.

WIMMER, H. (1980). Children's understanding of stories: Assimilation by a general schemata for actions or coordination of temporal relations. En F. Wilkening, J. Becker y T. Trabasso (comps.): Information integration by children. Hillsdale, N. J.: Erlbaum.

\section{Apéndice}

Ejemplos de narraciones

(Se ha respetado el texto de los cuentos, si bien se ha normalizado su ortografía y puntuación.)

Tipo 1

a

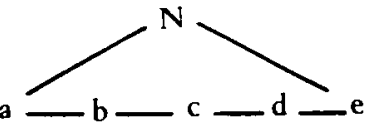

(Se trata, como puede verse en los ejemplos, de acciones yuxtapuestas sin llegar a engarzarse en un nudo común.)

\section{EJEMPLO ESCRITO}

Habia un gato que estaba a la puerta, había una abuela ciega; de pronto, el gato corrió muy rápido y colorin este cuento se acabó.

\section{EJEMPLO ORAL}

Era una vez una granja; y un día un señor se montó en el caballo y su amigo se fue a cazar, y cazó un lobo y después su amigo volvió y se bajó del caballo, y le preguntó qué había cazado y le dijo que un lobo, y después lo frieron y lo pusieron en un plato y empezaron a comérselo. Cuando ya se lo comieron se fueron a trabajar, y después éste fue a fregar los platos; cuando fregó, se fue a hacer la compra, y trajo un conejo para asar. Cuando ya lo asó lo puso aquí; después su amigo volvió, se bajó del caballo y se puso otra vez a cenar; cuando terminó se acostaron este fue a fregar y después se acostaron, y éste ya se acostó. 
Tipo 2

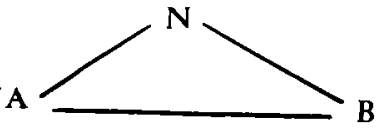

(Se trata de narraciones con un solo nudo, pero descrito muy simplemente sin acciones intermedias.)

\section{EJEMPLO ESCRITO}

Era una vez un gatito y un niño que vivía en una casa, y una vez pasó un ratón y una rata, y llamó la rata «tamtam», abrió la puerta el niño y dijo la rata: « ¿podemos vivir con vosotros?» y dijo el niño: «sí», y vivieron felices.

Tipo 3

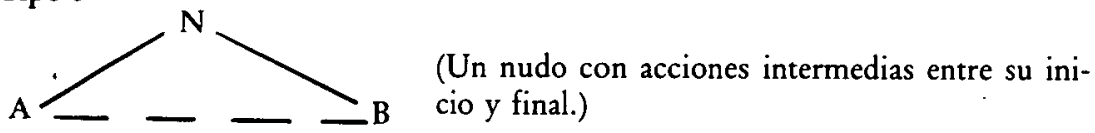

\section{EJEMPLO ORAL}

Era una vez una niña que le iba a llevar comida a su mamá, luego pasó un hombre y le dijo: "¿a dónde vas?», le dijo: «a llevar comida a mi mamá", y entonces dijo el señor: "yo te llevaré, que si no te perderás». Y luego llamaron a la puerta de su mamá y abrió un oso, y entonces la niña se acercó al oso y luego le empezó a tocar, pero el oso le empezó a tocar a la niña y luego la niña se sentó y la agarró de la mano y se fueron juntos a casa y ya se ha acabado.

Tipo 4
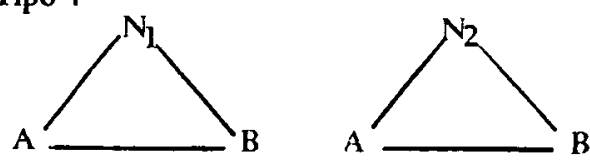

(Dos nudos simples relacionados.)

\section{EJEMPLO ESCRITO}

Era una vez una niña que se encontró un gato asustado y lo llevó a su casa, y lo cuidó y estaba muy contento; y la niña tenía un ratón en su casa, entonces el gato se escondió y comió al ratón y este cuento se acabó.

Tipo 5
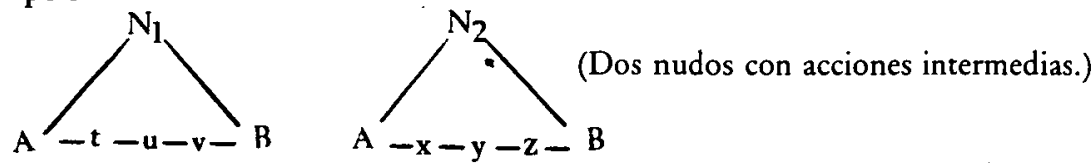

\section{EJEMPLO ORAL}

Pues el cuento empieza que había un niño que era príncipe de un país, y su padre era muy pobre y siempre le daba de comer lo mismo, y al niño no le gustaba 
la comida. Y un dia se fue a cazar y cazó muchos animales, y ya comía lo que le gustaba. Y luego un día se tenía que casar porque se había hecho mayor, y encontró a una chica muy guapa y le dijó: "qué guapa eres, cásate conmigo». Pero ella lo vio pobre porque se había puesto un disfraz de pobre y le dijo: «no, no, no". Luego andando, andando vio a otra que también era pobre y le dijo: «cásate conmigo" y ella le dijo que sí. Se casaron y luego se vistió bien y era joven y rico.

\section{Tipo 6}

(No se han registrado ejemplos.)

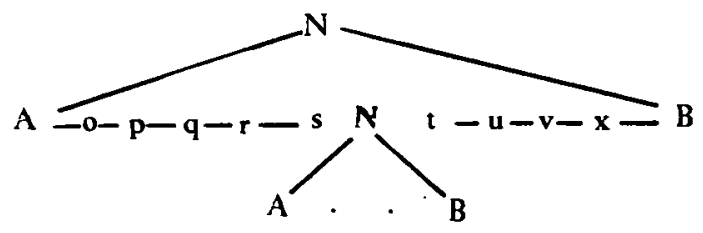

\section{Tipo 7}

(No se han encontrado ejemplos.)

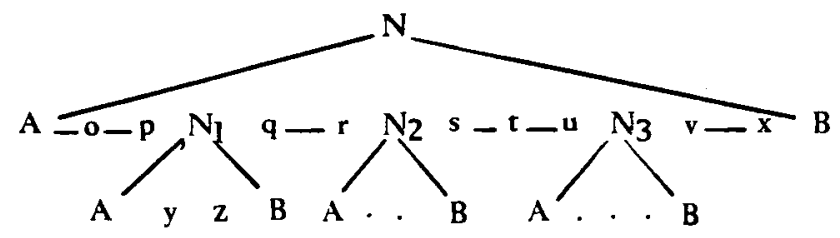

